\title{
The Exact Distribution of the Number of Vertices of a Random Convex Chain
}

\author{
Christian Buchta
}

Vienna, Preprint ESI 1886 (2006)

December 28, 2006

Supported by the Austrian Federal Ministry of Education, Science and Culture Available via http://www.esi.ac.at 


\title{
The Exact Distribution of the Number of Vertices of a Random Convex Chain
}

\author{
Christian Buchta \\ Universität Salzburg, Fachbereich Mathematik \\ Hellbrunner Straße 34, A-5020 Salzburg, Austria \\ For the sixty-fifth birthday of Rolf Schneider
}

\begin{abstract}
Assume that $n$ points $P_{1}, \ldots, P_{n}$ are distributed independently and uniformly in the triangle with vertices $(0,1),(0,0)$, and $(1,0)$. Consider the convex hull of $(0,1), P_{1}, \ldots, P_{n}$, and $(1,0)$. The vertices of the convex hull form a convex chain. Let $p_{k}^{(n)}$ be the probability that the convex chain consists - apart from the points $(0,1)$ and $(1,0)$ - of exactly $k$ of the points $P_{1}, \ldots, P_{n}$. Bárány, Rote, Steiger, and Zhang [3] proved that $p_{n}^{(n)}=2^{n} /[n !(n+1) !]$. We determine for $k=1, \ldots, n-1$ the values of $p_{k}^{(n)}$ and thus obtain the distribution of the number of vertices of a random convex chain. Knowing this distribution provides the key to the answer of some long-standing questions in geometrical probability.
\end{abstract}

Mathematics Subject Classification (2000) 52A22 60D05

Key Words: Random convex chain, random polygon, exact distribution, number of vertices, Sylvester's problem, geometrical probability.

\section{Introduction}

The question of determining the distribution of the number of vertices of a random convex chain arises in the context of the following old and unexpectedly difficult problem.

Assume that $n$ points are chosen independently and according to the uniform distribution from some compact convex set $C$ like, e.g., a square or a triangle. Consider the convex hull of the randomly chosen points. What is the probability $p_{k}^{(n)}(C)$ that the convex hull has exactly $k$ vertices? In other words: What is the distribution of the number $N_{n}(C)$ of vertices of the convex hull? 
For $n=4$ points (for smaller $n$ everything is trivial) the problem was raised by Sylvester and answered by Woolhouse in 1867: For $S$ a square and $T$ a triangle $p_{3}^{(4)}(S)=\frac{11}{36}, p_{3}^{(4)}(T)=\frac{1}{3}$, and, correspondingly, $p_{4}^{(4)}(S)=\frac{25}{36}$, $p_{4}^{(4)}(T)=\frac{2}{3}$. On the one hand, the values for $p_{3}^{(4)}(S)$ and $p_{3}^{(4)}(T)$ were extended to

$$
p_{3}^{(n)}(S)=\frac{1}{2^{n-5} n(n-1)} \sum_{j=1}^{n-1} \frac{1}{j}
$$

by Reed [9] and Henze [8] (whereat the latter corrected the result of the former) and to

$$
p_{3}^{(n)}(T)=\frac{12}{(n-1)^{2}(2 n-1)}+\frac{2}{((n-2) !)^{2}(2 n-1)} \sum_{j=0}^{n-3}(j !(n-3-j) !)^{2}
$$

independently by Reed [9] and Alagar [1]. On the other hand, the values for $p_{4}^{(4)}(S)$ and $p_{4}^{(4)}(T)$ were extended to

$$
p_{n}^{(n)}(S)=\left(\frac{1}{n !}\left(\begin{array}{c}
2 n-2 \\
n-1
\end{array}\right)\right)^{2}, \quad p_{n}^{(n)}(T)=\frac{2^{n}(3 n-3) !}{((n-1) !)^{3}(2 n) !}
$$

by Valtr in [10] and [11], respectively.

As long as the result of the present paper is not available, $p_{k}^{(n)}(C)$ is unknown for $k=4, \ldots, n-1$ for any convex set $C$ if $n \geq 7$. (The values $p_{4}^{(5)}(S)=\frac{5}{9}$ and $p_{4}^{(5)}(T)=\frac{5}{9}$ are immediate consequences of the abovementioned results. In fact, they had been derived in [5] before the work of Valtr. The values $p_{4}^{(6)}(S)=\frac{1307}{3600}, p_{4}^{(6)}(T)=\frac{119}{300}, p_{5}^{(6)}(S)=\frac{343}{720}$, and $p_{5}^{(6)}(T)=\frac{13}{30}$ follow from the above-mentioned results together with the expected number of vertices of the convex hull, which was determined by the author in [4] for general $n$; also cf. Section 5.2 of [7].)

Now assume that $C$ is a convex polygon. Consider two adjacent edges and those two of $n$ points chosen at random from $C$ which have the smallest distances to these edges. (The possibility that one and the same point has the smallest distances to both edges has to be dealt with separately.) Clearly, both points are vertices of the convex hull of the chosen points. The knowledge of the number of points which are also vertices of the convex hull and are situated - in an obvious sense - "between" the two points will give rise to the knowledge of the total number $N_{n}(C)$ of vertices of the convex hull.

Associating - in regard to affine invariance - the two points with the smallest distances to the considered adjacent edges with the points $(0,1)$ 
and $(1,0)$, associating the two lines which pass through one of the points and are parallel to the respective edges with the co-ordinate axes, and hence associating their intersection point with the point $(0,0)$, we are led to the question answered in the next section. This question is of independent interest. What will follow in the present paper is a complete answer to this question.

It will be shown in a separate paper how the achieved answer can be used to determine the exact distribution of the number $N_{n}(C)$ of vertices of the convex hull of $n$ random points in $C$. The details are intricate. Here we only state a resulting formula for illustration: In the case of a triangle $T$ it is well known that the expected value of $N_{n}(T)$ is given by

$$
E N_{n}(T)=2 \sum_{k=1}^{n-1} \frac{1}{k}
$$

cf. [4]. The corresponding expression for the variance will be shown to be

$$
\operatorname{var} N_{n}(T)=\frac{10}{9} \sum_{k=1}^{n-1} \frac{1}{k}-\frac{4}{3} \sum_{k=1}^{n-1} \frac{1}{k^{2}} .
$$

The result of the present paper was announced in [6], where a further application is described. It is beyond the scope of this article to give a survey on related results. Two recent papers by Vu [12] and by Bárány and Reitzner [2] contain many references and describe the concern of the present article in a broad context; see in particular the last paragraph on p. 1285 of $[12]$.

\section{Main result and its proof}

TheOREM. Assume that $n$ points $P_{1}, \ldots, P_{n}$ are distributed independently and uniformly in the triangle with vertices $(0,1),(0,0)$, and $(1,0)$. Let $p_{k}^{(n)}$ be the probability that exactly $k$ of the points $P_{1}, \ldots, P_{n}$ are vertices of the convex hull of $(0,1), P_{1}, \ldots, P_{n}$, and $(1,0)$. Then

$$
\begin{aligned}
p_{k}^{(n)}=2^{k} \sum & \frac{1}{i_{1}\left(i_{1}+i_{2}\right) \ldots\left(i_{1}+\ldots+i_{k}\right)} \\
& \cdot \frac{i_{1} \ldots i_{k}}{\left(i_{1}+1\right)\left(i_{1}+i_{2}+1\right) \ldots\left(i_{1}+\ldots+i_{k}+1\right)},
\end{aligned}
$$

where the sum is taken over all $i_{1}, \ldots, i_{k} \in \mathbb{N}$ such that $i_{1}+\ldots+i_{k}=n$. 
Proof. We are going to determine the probability $p^{(n)}\left(P_{1}, \ldots, P_{k}\right)$ that those of the points $P_{1}, \ldots, P_{n}$ which are vertices of the convex hull of $(0,1), P_{1}, \ldots, P_{n}$, and $(1,0)$ are just the points $P_{1}, \ldots, P_{k}$ in such a way that the point $P_{1}$ is the vertex closest to $(0,1)$, the point $P_{2}$ is the succeeding vertex in counter-clockwise direction, etc., and finally the point $P_{k}$ is the vertex closest to $(1,0)$. As soon as $p^{(n)}\left(P_{1}, \ldots, P_{k}\right)$ has been obtained, the required probability $p_{k}^{(n)}$ follows immediately: Since the points $P_{1}, \ldots, P_{n}$ are independently and uniformly distributed, they are pairwise different and different from $(0,1)$ and from $(1,0)$ with probability one. Clearly, given $n$ different points, there are $\frac{n !}{(n-k) !}$ possibilities to specify $k$ of them in a certain order. Hence, as the points $P_{1}, \ldots, P_{n}$ are identically distributed,

$$
p_{k}^{(n)}=\frac{n !}{(n-k) !} p^{(n)}\left(P_{1}, \ldots, P_{k}\right) .
$$

Assume that the point $(0,1), j-1$ points $P_{1}, \ldots, P_{j-1}$, which are contained in the triangle with vertices $(0,1),(0,0)$, and $(1,0)$, and the point $(1,0)$ form a convex chain in counter-clockwise direction, i.e. the vertices of the convex hull of these points - taken counter-clockwise starting with $(0,1)$ - are just the points in the given order. A further point in the triangle, $P_{j}$ say, extends the convex chain $(0,1), P_{1}, \ldots, P_{j-1},(1,0)$ to a convex chain $(0,1), P_{1}, \ldots, P_{j},(1,0)$ if and only if $P_{j}$ lies "above" the line through $P_{j-2}$ and $P_{j-1}$ and "below" the line through $P_{j-1}$ and $(1,0)$.

Denoting the co-ordinates of $P_{j}, j=1,2, \ldots$, by $a_{j}$ and $b_{j}$, and defining $a_{0}=0, b_{0}=1$, the first condition is fulfilled if and only if

$$
a_{j-2}\left(b_{j-1}-b_{j}\right)+a_{j-1}\left(b_{j}-b_{j-2}\right)+a_{j}\left(b_{j-2}-b_{j-1}\right) \geq 0,
$$

or, equivalently, if and only if

$$
a_{j} \geq \frac{a_{j-2}\left(b_{j}-b_{j-1}\right)+a_{j-1}\left(b_{j-2}-b_{j}\right)}{b_{j-2}-b_{j-1}} .
$$

The second condition is fulfilled if and only if

$$
a_{j-1} b_{j}-a_{j} b_{j-1}+b_{j-1}-b_{j} \geq 0,
$$

or, equivalently, if and only if

$$
a_{j} \leq \frac{a_{j-1} b_{j}+b_{j-1}-b_{j}}{b_{j-1}} .
$$


Thus we see that the co-ordinates $a_{j}$ and $b_{j}$ of the point $P_{j}$ have to fulfill the restrictions

$$
0 \leq b_{j} \leq b_{j-1}
$$

and

$$
\begin{array}{r}
\frac{a_{j-2}\left(b_{j}-b_{j-1}\right)+}{b_{j-2}-} a_{j-1}\left(b_{j-2}-b_{j}\right) \\
\leq \frac{a_{j-1} b_{j}+b_{j-1}-b_{j}}{b_{j-1}}
\end{array}
$$

The inequality ( 1 ) holds for $j \geq 2$; for $j=1$ there is no restriction of this type, just $a_{1} \geq 0$. The inequality (2) holds for $j \geq 1$; for $j=1$ it reduces to $a_{1} \leq 1-b_{1}$.

Once the points $(0,1), P_{1}, \ldots, P_{k}$, and $(1,0)$ form a convex chain, the convex hull of these points consists of the $k$ triangles which have two consecutive points of the chain different from $(1,0)$ and the point $(1,0)$ as vertices. The area of the triangle with vertices $P_{j-1}, P_{j}$, and $(1,0)$, where $P_{0}=(0,1)$, is given by

$$
\frac{1}{2}\left(a_{j-1} b_{j}-a_{j} b_{j-1}+b_{j-1}-b_{j}\right)=: \frac{1}{2} \Delta j ;
$$

the area of the convex hull of $(0,1), P_{1}, \ldots, P_{k}$, and $(1,0)$ therefore by $\frac{1}{2}\left(\Delta_{1}+\right.$ $\left.\ldots+\Delta_{k}\right)$. Since the area of the triangle with vertices $(0,1),(0,0)$, and $(1,0)$ is $\frac{1}{2}$, the probability that any of the random points $P_{k+1}, \ldots, P_{n}$ falls into the convex hull of the fixed convex chain $(0,1), P_{1}, \ldots, P_{k}$, and $(1,0)$ consequently equals $\Delta_{1}+\ldots+\Delta_{k}$. The probability that all $n-k$ random points fall into the convex hull is then just

$$
\left(\Delta_{1}+\ldots+\Delta_{k}\right)^{n-k}=\sum_{j_{1}+\ldots+j_{k}=n-k} \frac{(n-k) !}{j_{1} ! \ldots j_{k} !} \Delta_{1}^{j_{1}} \ldots \Delta_{k}^{j_{k}} .
$$

Integration of the sum on the right hand side with respect to the co-ordinates $a_{1}, \ldots, a_{k}$ and $b_{1}, \ldots, b_{k}$ of the points $P_{1}, \ldots, P_{k}$ will now imply the required value of $p^{(n)}\left(P_{1}, \ldots, P_{k}\right)$.

The main difficulty in evaluating the $2 k$-fold integral are the involved bounds (1) and (2) of the variables $a_{2}, \ldots, a_{k}$. First notice that $\Delta_{j}$ is a function of $a_{j-1}$ and $a_{j}$, and therefore, among $\Delta_{1}, \ldots, \Delta_{j}$, only $\Delta_{j}$ depends on $a_{j}$. In view of this situation it is crucial to observe that the integration of a power of $\Delta_{j}$ with respect to $a_{j}$ results in a power of $\Delta_{j-1}$ times a factor which does not depend on $a_{1}, \ldots, a_{j-1}$ : With lower and upper bound given 
by (1) and (2), respectively, a straight forward calculation shows that

$$
\begin{aligned}
& \int \Delta_{j}^{r} d a_{j} \\
& =\frac{1}{r+1} \frac{1}{b_{j-1}}\left(\frac{b_{j-1}-b_{j}}{b_{j-2}-b_{j-1}}\left(a_{j-2} b_{j-1}-a_{j-1} b_{j-2}+b_{j-2}-b_{j-1}\right)\right)^{r+1} \\
& =\frac{1}{r+1} \frac{1}{b_{j-1}}\left(\frac{b_{j-1}-b_{j}}{b_{j-2}-b_{j-1}} \Delta_{j-1}\right)^{r+1} .
\end{aligned}
$$

Successive exploitation of this observation for $j=k, k-1, \ldots, 2$ (recall in the case $j=2$ that $b_{0}=1$ ) together with

$$
\int_{0}^{1-b_{1}} \Delta_{1}^{r} d a_{1}=\frac{1}{r+1}\left(1-b_{1}\right)^{r+1}
$$

yields

$$
\begin{aligned}
\int & \Delta_{1}^{j_{1}}\left(\int \Delta_{2}^{j_{2}} \ldots \int \Delta_{k-1}^{j_{k-1}}\left(\int \Delta_{k}^{j_{k}} d a_{k}\right) d a_{k-1} \ldots d a_{2}\right) d a_{1} \\
= & \frac{1}{j_{k}+1} \frac{1}{b_{k-1}}\left(\frac{b_{k-1}-b_{k}}{b_{k-2}-b_{k-1}}\right)^{j_{k}+1} \\
& \cdot \frac{1}{j_{k-1}+j_{k}+2} \frac{1}{b_{k-2}}\left(\frac{b_{k-2}-b_{k-1}}{b_{k-3}-b_{k-2}}\right)^{j_{k-1}+j_{k}+2} \\
& \cdot \ldots \\
& \cdot \frac{1}{j_{2}+\ldots+j_{k}+k-1} \frac{1}{b_{1}}\left(\frac{b_{1}-b_{2}}{1-b_{1}}\right)^{j_{2}+\ldots+j_{k}+k-1} \\
& \cdot \frac{1}{j_{1}+\ldots+j_{k}+k}\left(1-b_{1}\right)^{j_{1}+\ldots+j_{k}+k} \\
= & \frac{1}{\left(j_{k}+1\right)\left(j_{k-1}+j_{k}+2\right) \ldots\left(j_{2}+\ldots+j_{k}+k-1\right)\left(j_{1}+\ldots+j_{k}+k\right)} \\
& \cdot \frac{\left(b_{k-1}-b_{k}\right)^{j_{k}+1}\left(b_{k-2}-b_{k-1}\right)^{j_{k-1}+1} \ldots\left(b_{1}-b_{2}\right)^{j_{2}+1}\left(1-b_{1}\right)^{j_{1}+1}}{b_{k-1} b_{k-2} \ldots b_{1}} .
\end{aligned}
$$

Considering the bounds $0 \leq b_{j} \leq b_{j-1}$ (with $b_{0}=1$ ), integration with respect to $b_{j}$ for $j=k, k-1, \ldots, 1$ reduces to a repeated application of the 
elementary identity

$$
\int_{0}^{b_{j-1}} b_{j}^{p}\left(b_{j-1}-b_{j}\right)^{q} d b_{j}=\frac{p ! q !}{(p+q+1) !} b_{j-1}^{p+q+1} .
$$

Thus we see that

$$
\begin{aligned}
& \int \ldots \int \Delta_{1}^{j_{1}} \ldots \Delta_{k}^{j_{k}} d a_{k} \ldots d a_{1} d b_{k} \ldots d b_{1} \\
&=\frac{1}{\left(j_{k}+1\right)\left(j_{k-1}+j_{k}+2\right) \ldots\left(j_{2}+\ldots+j_{k}+k-1\right)\left(j_{1}+\ldots+j_{k}+k\right)} \\
& \cdot \frac{0 !\left(j_{k}+1\right) !}{\left(j_{k}+2\right) !} \frac{\left(j_{k}+1\right) !\left(j_{k-1}+1\right) !}{\left(j_{k-1}+j_{k}+3\right) !} \frac{\left(j_{k-1}+j_{k}+2\right) !\left(j_{k-2}+1\right) !}{\left(j_{k-2}+j_{k-1}+j_{k}+4\right) !} \ldots \\
& \cdot \frac{\left(j_{3}+\ldots+j_{k}+k-2\right) !\left(j_{2}+1\right) !}{\left(j_{2}+\ldots+j_{k}+k\right) !} \frac{\left(j_{2}+\ldots+j_{k}+k-1\right) !\left(j_{1}+1\right) !}{\left(j_{1}+\ldots+j_{k}+k+1\right) !} \\
&= \frac{1}{n !} \frac{1}{\left(j_{k}+1\right)\left(j_{k-1}+j_{k}+2\right) \ldots\left(j_{2}+\ldots+j_{k}+k-1\right)\left(j_{1}+\ldots+j_{k}+k\right)} \\
& \cdot \frac{\left(j_{k}+1\right) !\left(j_{k-1}+1\right) ! \ldots\left(j_{2}+1\right) !\left(j_{1}+1\right) !}{\left(j_{k}+2\right)\left(j_{k-1}+j_{k}+3\right) \ldots\left(j_{2}+\ldots+j_{k}+k\right)\left(j_{1}+\ldots+j_{k}+k+1\right)}
\end{aligned}
$$

where the last sign of equality is justified by $j_{1}+\ldots+j_{k}=n-k$. Taking into account that the triangle with vertices $(0,1),(0,0)$, and $(1,0)$, in which the points $P_{1}, \ldots, P_{k}$ are uniformly distributed, has area $\frac{1}{2}$, we arrive at

$$
\begin{aligned}
p^{(n)}( & \left.P_{1}, \ldots, P_{k}\right) \\
= & \int \ldots \int\left(\Delta_{1}+\ldots+\Delta_{k}\right)^{n-k} \frac{d a_{1} d b_{1}}{\frac{1}{2}} \ldots \frac{d a_{k} d b_{k}}{\frac{1}{2}} \\
= & 2^{k} \sum_{j_{1}+\ldots+j_{k}=n-k} \frac{(n-k) !}{j_{1} ! \ldots j_{k} !} \frac{1}{n !} \frac{1}{\left(j_{k}+1\right)\left(j_{k-1}+j_{k}+2\right) \ldots\left(j_{1}+\ldots+j_{k}+k\right)} \\
& \cdot \frac{\left(j_{k}+1\right) !\left(j_{k-1}+1\right) ! \ldots\left(j_{1}+1\right) !}{\left(j_{k}+2\right)\left(j_{k-1}+j_{k}+3\right) \ldots\left(j_{1}+\ldots+j_{k}+k+1\right)} .
\end{aligned}
$$

Recalling that $p_{k}^{(n)}=\frac{n !}{(n-k) !} p^{(n)}\left(P_{1}, \ldots, P_{k}\right)$ and putting $j_{k}+1=: i_{1}, j_{k-1}+$ 
$1=: i_{2}, \ldots, j_{1}+1=: i_{k}$ we finally find that

$$
\begin{aligned}
p_{k}^{(n)}=2^{k} \sum_{i_{1}+\ldots+i_{k}=n} & \frac{1}{i_{1}\left(i_{1}+i_{2}\right) \ldots\left(i_{1}+\ldots+i_{k}\right)} \\
& \cdot \frac{i_{1} \ldots i_{k}}{\left(i_{1}+1\right)\left(i_{1}+i_{2}+1\right) \ldots\left(i_{1}+\ldots+i_{k}+1\right)},
\end{aligned}
$$

where the integers $i_{1}, \ldots, i_{k}$ are at least one.

\section{Evaluation of the sum in the Theorem}

Fixing the difference between $n$ and $k$, the sum in the Theorem can easily be evaluated by elementary and straight forward calculations:

When $k=n$, the equation $i_{1}+\ldots+i_{n}=n$ is fulfilled if and only if $i_{1}=\ldots=i_{n}=1$. Hence the sum representing $p_{n}^{(n)}$ consists of a single summand, and we immediately see that

$$
p_{n}^{(n)}=\frac{2^{n}}{n !(n+1) !}
$$

This is the result established by Bárány, Rote, Steiger, and Zhang [3]. (Compared to Theorem 1 in [3] the factor $2^{n}$ arises here since we consider a triangle instead of a square. Clearly, the event that $n$ points chosen independently and uniformly from the square with vertices $(0,1),(0,0),(1,0)$, and $(1,1)$ fall into the triangle with vertices $(0,1),(0,0)$, and $(1,0)$ has probability $1 / 2^{n}$.)

When $k=n-1$, the equation $i_{1}+\ldots+i_{n-1}=n$ is fulfilled if and only if one of the entries $i_{1}, \ldots, i_{n-1}$ is 2 , whereas all other entries are 1 . Taking into account that the common denominator of the arising summands is $n !(n+1)$ !, we immediately see that

$$
p_{n-1}^{(n)}=2^{n-1} \frac{2}{n !(n+1) !} \sum_{m=2}^{n}(m-1) m=\frac{2^{n}}{3 n !(n-2) !}
$$

since

$$
\sum_{m=2}^{n}(m-1) m=\frac{1}{3}(n-1) n(n+1) .
$$

When $k=n-2$, the equation $i_{1}+\ldots+i_{n-2}=n$ is fulfilled in the following two cases: 
(i) One of the entries $i_{1}, \ldots, i_{n-2}$ is 3 , whereas all other entries are 1 .

(ii) Two of the entries $i_{1}, \ldots, i_{n-2}$ are 2 , whereas all other entries are 1 .

The contribution to $p_{n-2}^{(n)}$ corresponding to the first case is given by

$$
\begin{aligned}
{ }^{(i)} p_{n-2}^{(n)} & =2^{n-2} \frac{3}{n !(n+1) !} \sum_{m=3}^{n}(m-2)(m-1)^{2} m \\
& =\frac{3 \cdot 2^{n-3}(2 n-1)}{5 n !(n-3) !}
\end{aligned}
$$

since

$$
\sum_{m=3}^{n}(m-2)(m-1)^{2} m=\frac{1}{10}(n-2)(n-1) n(n+1)(2 n-1) .
$$

The contribution to $p_{n-2}^{(n)}$ corresponding to the second case is given by

$$
\begin{aligned}
{ }^{(i i)} p_{n-2}^{(n)} & =2^{n-2} \frac{2.2}{n !(n+1) !} \sum_{m=4}^{n}\left(\sum_{j=2}^{m-2}(j-1) j\right)(m-1) m \\
& =\frac{2^{n-1}(5 n-2)}{45 n !(n-4) !}
\end{aligned}
$$

since the sum with respect to $j$ yields $\frac{1}{3}(m-3)(m-2)(m-1)$ and

$$
\sum_{m=4}^{n}(m-3)(m-2)(m-1)^{2} m=\frac{1}{30}(n-3)(n-2)(n-1) n(n+1)(5 n-2) .
$$

Therefore

$$
p_{n-2}^{(n)}={ }^{(i)} p_{n-2}^{(n)}+{ }^{(i i)} p_{n-2}^{(n)}=\frac{2^{n-3}\left(20 n^{2}-14 n-3\right)}{45 n !(n-3) !} .
$$

Now it is clear how to proceed when $n-k=3,4, \ldots$. We see that the evaluation of the sum in the Theorem for a fixed difference between $n$ and $k$ reduces to the computation of the sums

$$
\sum_{m=1}^{n} m^{p}, \quad p=1,2, \ldots
$$


For fixed $k$ the sum in the Theorem can be written in terms of harmonic numbers and iterated harmonic numbers. We note that

$$
\begin{gathered}
p_{1}^{(n)}=\frac{2}{n+1}, \quad p_{2}^{(n)}=\frac{4}{n} H_{n}-\frac{8}{n+1}, \\
p_{3}^{(n)}=\frac{8}{n+1} H_{n}^{(2)}-\frac{24}{n} H_{n}+\frac{40}{n+1},
\end{gathered}
$$

where

$$
H_{n}=\sum_{j=1}^{n} \frac{1}{j}, \quad H_{n}^{(2)}=\sum_{m=1}^{n} \frac{1}{m} \sum_{j=1}^{m} \frac{1}{j} .
$$

Similar expressions can be obtained for $k=4,5, \ldots$.

\section{References}

[1] V. S. Alagar, On the distribution of a random triangle. J. Appl. Prob. 14 (1977), 284-297.

[2] I. Bárány, M. Reitzner, Random polytopes. Manuscript.

[3] I. Bárány, G. Rote, W. Steiger, and C.-H. Zhang, A central limit theorem for convex chains in the square. Discrete Comput. Geom. 23 (2000), 35-50.

[4] C. Buchta, Zufallspolygone in konvexen Vielecken. J. Reine Angew. Math. 347 (1984), 212-220.

[5] C. Buchta, On a conjecture of R. E. Miles about the convex hull of random points. Monatsh. Math. 102 (1986), 91-102.

[6] C. Buchta, On the distribution of the number of vertices of random polygon. Anz. Österr. Akad. Wiss., Math.-Naturwiss. Kl., Abt. II, 139 (2003), 17-19.

[7] C. Buchta, An identity relating moments of functionals of convex hulls. Discrete Comput. Geom. 33 (2005), 125-142.

[8] N. Henze, Random triangles in convex regions. J. Appl. Prob. 20 (1983), 111-125.

[9] W. J. Reed, Random points in a simplex. Pacific J. Math. 54 (1974), 183-198. 
[10] P. Valtr, Probability that $n$ random points are in convex position. Discrete Comput. Geom. 13 (1995), 637-643.

[11] P. Valtr, The probability that $n$ random points in a triangle are in convex position. Combinatorica 16 (1996), 567-573.

[12] V. H. Vu, Sharp concentration of random polytopes. Geom. Funct. Anal. 15 (2005), 1284-1318. 